\title{
Monte Carlo Simulation of Ferroelectric Domain Structure: Electrostatic and Elastic Strain Energy Contributions
}

\author{
B.G. Potter, Jr., B.A. Tuttle, and V. Tikare
}

Sandia National Laboratories

Albuquerque, NM 87185-1405

\begin{abstract}
A lattice-Monte Carlo approach was developed to simulate ferroelectric domain behavior. The model utilizes a Hamiltonian for the total energy that includes electrostatic terms (involving dipole-dipole interactions, local polarization gradients, and applied electric field), and elastic strain energy. The contributions of these energy components to the domain structure and to the overall applied field response of the system were examined. In general, the model exhibited domain structure characteristics consistent with those observed in a tetragonally distorted ferroelectric. Good qualitative agreement between the appearance of simulated electrical hysteresis loops and those characteristic of real ferroelectric materials was found.
\end{abstract}

\section{INTRODUCTION}

The electrical, mechanical and optical behavior of a ferroelectric is dictated by the morphology and reorientation dynamics of domains, regions consisting of likeoriented spontaneous polarization. The ferroelectric attempts to attain a domain configuration that minimizes the total energy while satisfying both electrostatic and mechanical boundary conditions. The interplay between domain configuration, microstructure, and service conditions (e.g. temperature, applied field, pressure) dictate the engineering performance of a ferroelectric. Development of a computational approach to simulate the domain structure of a ferroelectric would enhance a broader model to predict the mechanical and electrical response of a ferroelectric. This capability could be used to guide the processing and utilization of ferroelectric ceramics in various applications to optimize performance and evaluate aging effects.

Numerical simulations of ferroelectrics and their properties have been the focus of numerous research efforts. Techniques including ab initio calculations [1-3], the solution of the Landau-Ginzburg energy relations [4-6], and Potts-based Monte Carlo simulations $[7,8]$ have been pursued. The present work develops a lattice-based Monte Carlo simulation technique for the prediction of ferroelectric domain configurations. The model is two-dimensional and, rather than relying on the typical Potts-type Hamiltonian to evaluate neighboring lattice site interactions, the present 


\section{DISCLAIMER}

This report was prepared as an account of work sponsored by an agency of the United States Government. Neither the United States Government nor any agency thereof, nor any of their employees, make any warranty, express or implied, or assumes any legal liability or responsibility for the accuracy, completeness, or usefulness of any information, apparatus, product, or process disclosed, or represents that its use would not infringe privately owned rights. Reference herein to any specific commercial product, process, or service by trade name, trademark, manufacturer, or otherwise does not necessarily constitute or imply its endorsement, recommendation, or favoring by the United States Government or any agency thereof. The views and opinions of authors expressed herein do not necessarily state or reflect those of the United States Government or any agency thereof. 


\section{DISCLAIMER}

Portions of this document may be illegible in electronic image products. Images are produced from the best available original document. 
approach utilizes potentials based on electrostatic and mechanical phenomena that occur in ferroelectrics. As we will discuss below, these longer range interactions produce domain morphologies and relative orientations of dipoles across domain boundaries that are consistent with those observed experimentally.

The present work will describe the model and the influence of the electrostatic and elastic strain energy terms used on the evolution of domain assemblage. The response of the dipole ensemble to an oscillating applied field will also be examined through the simulation of electrical hysteresis loops.

\section{MODEL DESCRIPTION}

\section{Crystal and Domain Representation}

In the current lattice-based Monte Carlo (MC) simulation, space is discretized into a two-dimensional, square array, or grid, of lattice points. Each lattice point is assigned the orientation and magnitude of the local, spontaneous dipole moment associated with a single ferroelectric unit cell.

A tetragonal lattice distortion is assumed to describe the formation of permanent dipoles in the ferroelectric material. Two orthogonal basis vectors (twodimensions) are therefore used to describe the local dipole orientation. Four dipole orientations are allowed within the plane defined by the basis vectors. The simulation is further limited to a single crystal orientation with dipole moment basis vectors aligned with the principal axes of the square lattice used to discretize space. Allowed dipole orientations are therefore up, down, left and right.

While there is no inherent restriction on the relative orientation and symmetry used for the simulation lattice, the alignment of the simulation lattice basis vectors with those used to represent the dipole moment is consistent with a one-to-one correspondence between a simulation lattice site and a single ferroelectric unit cell and the assumption that crystallographic lattice coherence is maintained through the cubic to tetragonal phase transformation. This choice of lattice and dipole orientation symmetries thus enables the simulation to clearly exhibit the general domain wall orientations and the relative orientations of dipoles in adjacent domains that are anticipated in a tetragonal ferroelectric material (e.g. $180^{\circ}$ and $90^{\circ}$ domain walls).

The dipole moment magnitudes are assumed to be constant during the simulation. In most cases, the local dipole orientations were randomly assigned to lattice points to furnish a starting configuration. While the elastic strain energy is utilized in the model, the current implementation does not allow for motion of adjacent lattice sites with respect to one another, thus neglecting the influence of local displacements that describe the elastic strain field. 


\section{Interaction Potentials}

The present work attempts to introduce physically realistic interaction potentials based on the known electrostatic and elastic mechanical nature of the ferroelectric material associated with each lattice point. Since the lattice sites are associated with permanent dipole moments within the ferroelectric, a dipole-dipole potential energy expression has been introduced. Thus, the pairwise interaction potential energy between lattice points is given by the vector expression:

$$
E_{i j}=K \frac{\vec{P}_{i} \cdot \vec{P}_{j}-\left(3\left(\dot{P}_{i} \cdot \hat{n}\right)\left(\vec{P}_{j} \cdot \hat{n}\right)\right)}{r^{3}}
$$

where: $\mathrm{P}=$ dipole moment vector at a given lattice site; $\mathrm{n}=$ unit vector connecting points $i$ and $j ; r=$ magnitude of the separation vector between $i$ and $j ; K=$ scaling factor (contains the material permittivity).

The dipole-dipole interaction is truncated at a distance where the potential has dropped to $<2 \%$ of its magnitude at $r=1$ lattice unit. In addition, the truncation distance must also be less than one-half the size of the simulation box to avoid a dipole interacting with its image when using periodic boundary conditions (minimum image convention). A truncation distance of 6 lattice unit distances was utilized in the present study; larger truncation distances did not significantly alter the steady-state dipole configurations obtained or their dipole-dipole potential energy [9].

An potential term linked to the presence of polarization gradients is used to include an electrostatically based domain wall energy. The expression is adapted from that used by $\mathrm{Hu}$ and $\mathrm{Chen}[6]$. The potential energy density takes the form:

$$
D_{i}=-J\left\{\begin{array}{l}
\left(\frac{\Delta P_{x}}{\Delta x}\right)^{2}+\left(\frac{\Delta P_{y}}{\Delta y}\right)^{2}+\left(\frac{\Delta P_{x}}{\Delta x}\right)\left(\frac{\Delta P_{y}}{\Delta y}\right)+ \\
\left(\frac{\Delta P_{x}}{\Delta y}+\frac{\Delta P_{y}}{\Delta x}\right)^{2}+\left(\frac{\Delta P_{x}}{\Delta y}-\frac{\Delta P_{y}}{\Delta x}\right)^{2}
\end{array}\right\}
$$

where: $\mathrm{x}$ and $\mathrm{y}$ denote spatial variables, $\mathrm{P}$ is the local electric polarization, and $\mathrm{J}$ is a scaling factor for the domain wall interaction potential. Unless otherwise noted, the $\mathbf{J}$ factor in the present work was chosen to energetically favor a single domain configuration under periodic boundary conditions (infinite single crystal model) and a multi-domain structure under finite boundary conditions when using only electrostatic contributions to the total configuration energy.

An applied bias field was also included in the simulation. The potential energy of the dipole in the field is expressed as the dot product between the field vector and the dipole moment, as dictated by electrostatics. In the present study, the applied field 
is taken to be constant over the entire lattice without regard for electrostatic screening effects known to produce local modifications in the field strength. The field-related potential energy is therefore given as:

$$
E_{i}=-\alpha\left(P_{i} \cdot F\right)
$$

where: $\mathrm{P}=$ dipole moment, $\mathrm{F}=$ applied field, $\alpha=$ scaling factor.

Elastic strain energy at each step in the Monte Carlo simulation is calculated based on an analytical expression developed by Khachaturyan [10] for a multiphase, coherent mixture assuming a uniform, anisotropic elastic modulus in each phase. In this case, each dipole is associated with a portion of material that has undergone a phase transformation from cubic (paraelectric) to tetragonal (ferroelectric). The orientation of the dipole at a location in the simulation then gives the orientation of the local transformation strain field. In the present study, the simulation is therefore treated as a composite of two different material phases with the spatial distribution of these phases given by the dipole configuration. The total elastic strain energy for the ferroelectric body is given in terms of the elastic strain energy associated with the local transformations (term 1 in equation (4) below) and the concomitant relaxation of the surrounding material (terms 2 and 3 ).

$$
\begin{aligned}
E_{\text {elastic }}= & \frac{1}{2} V \sum_{p} \lambda_{i j k l} \varepsilon_{i j}^{o}(p) \varepsilon_{k l}^{o}(p) w_{p}-\frac{1}{2} V \sum_{p, q} \lambda_{i j k l} \varepsilon_{l j}^{o}(p) \varepsilon_{k l}^{o}(q) w_{p} w_{q} \\
& -\frac{1}{2} \sum_{p, q} \int \frac{d^{3} k}{(2 \pi)^{3}} \mathbf{n} \hat{\sigma}^{o}(p) \hat{\Omega}(\mathbf{n}) \hat{\sigma}^{o}(q) \mathbf{n} \Theta_{p}(\mathbf{k}) \Theta_{q}^{*}(\mathbf{k})
\end{aligned}
$$

where: $V=$ total volume, $\varepsilon^{0}(p)=$ transformation strain of phase $p, \sigma^{0}(p)=$ transformation stress of phase $\mathrm{p}, \lambda=$ elastic modulus tensor, $w_{p}=$ volume fraction of phase $p, \Theta_{p}(k)=$ Fourier transform of the "shape" function, $\Theta_{p}(r)$ (a spatial mapping of the pth phase), and $\Omega$ is a tensor whose inverse contains $\lambda$.

The relaxation energy is expressed in terms of homogeneous and heterogeneous strain components (terms 2 and 3 in equation (4), respectively) where the heterogeneous strain term is defined such that the total real-space displacement at the body edges is zero. All displacements associated with the body edges are contained in the homogeneous strain term.

Again, information regarding real-space displacements of the simulation lattice is not used in the current model. The strain energy calculated above is combined with the electrostatic energy to determine the total energy change associated with a given Monte Carlo move. 


\section{Simulation Units}

Electrostatic energies were calculated in reduced units with length defined by a simulation lattice constant (called a "lattice unit" (LU) ) and the dipole moment defined to be 1 charge-LU. Given the current assignment of a ferroelectric unit cell to each simulation lattice point, the $L U$ is therefore directly related to the crystallographic lattice constant. An energy unit (EU) is then equal to the potential energy obtained with two parallel dipole moments of unit magnitude separated by a vector of length one lattice unit oriented perpendicular to the dipole moments. Temperature is reported in terms of energy, i.e. kT, in EU's.

Elastic strain energies were calculated using elastic constants and transformation strains representative of $\mathrm{BaTiO}_{3}: \mathrm{C}_{11}=275 \times 10^{9} \mathrm{~N} / \mathrm{m}^{2}, \mathrm{C}_{12}=179 \mathrm{x}$ $10^{9} \mathrm{~N} / \mathrm{m}^{2}, \mathrm{C}_{44}=54.3 \times 10^{9} \mathrm{~N} / \mathrm{m}^{2}, \varepsilon_{11}=\varepsilon_{22}=-3.74 \times 10^{-3}, \varepsilon_{33}=6.23 \times 10^{-3}$. The results were converted to energy units (EU) to allow combination with electrostatic energy results to derive a total energy for the simulation.

The temporal unit is a Monte Carlo timestep (MCS). The MC timestep represents $\mathrm{mx} \mathrm{m} \mathrm{MC} \mathrm{move} \mathrm{attempts,} \mathrm{where} \mathrm{m}$ is the size of the simulation box. $50 \mathrm{x}$ 50 or $100 \times 100 \mathrm{LU}$ simulation sizes were typically utilized in this study. Where smaller ensembles are noted, the results are representative of larger simulations.

\section{Simulation Procedure}

A Monte Carlo move attempt involves the random selection of a lattice site followed by random reorientation of the dipole at this site. Move acceptance is determined using the Metropolis importance sampling algorithm [11] based on the energy change associated with the dipole reorientation and the simulation temperature.

Simulation temperatures were chosen to provide sufficient thermal energy to allow Monte Carlo move acceptance rates in the range 0.2 to 0.4 , thus favoring evolution of a steady-state dipole configuration within a reasonable computation time without significant randomization of the configuration due to thermal energy. A steady-state condition was defined when little or no further minimization in the ensemble's average total energy was observed with additional MC moves. Simulations were performed using either the electrostatic potential only or using both electrostatic and elastic strain energy contributions to the total energy. This allowed the effect of each energy term on the evolution of the dipole ensemble configuration to be studied. A more detailed evaluation of the specific effects of the individual electrostatic terms was undertaken in previous work [9].

After gaining insight into the relative impact of the different energy terms and simulation conditions on the evolution of the dipole configuration, the model was extended to allow the simulation of ferroelectric-type electrical hysteresis loops. In this case, the applied field value was changed by a known increment and the simulation was allowed to evolve from the configuration state developed at the previous field value. The net polarization component aligned with the applied field was calculated from the dipole configuration at each field magnitude. The "effective" field frequency was varied by changing the equilibration time allowed the system at a 

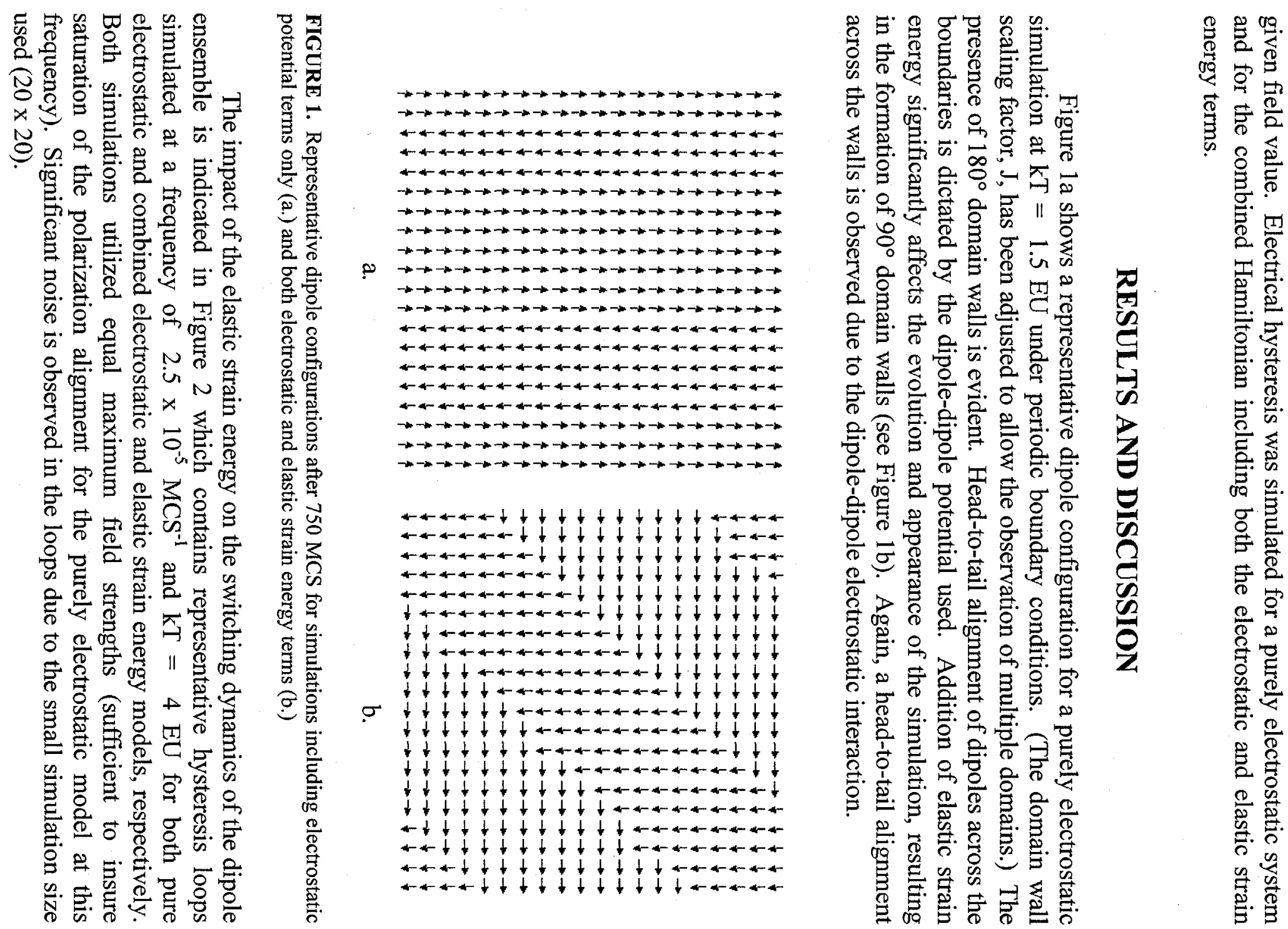


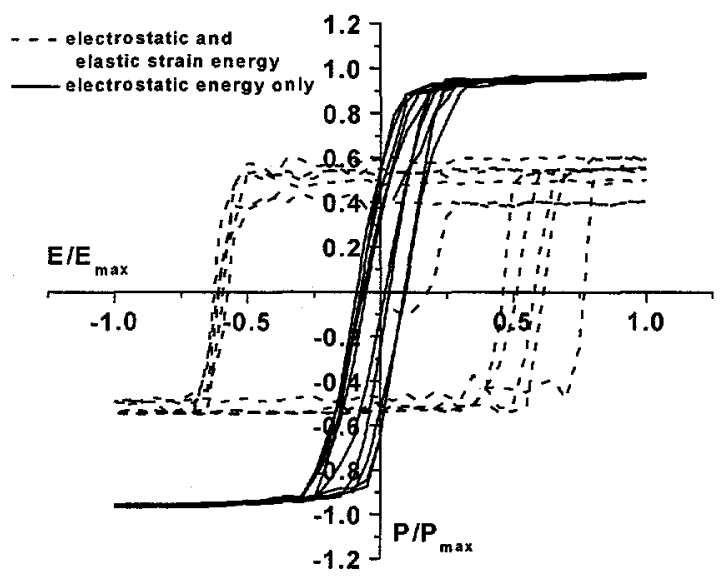

FIGURE 2. Representative electrical hysteresis loops simulated using the lattice-MC approach. The solid curve was obtained using only electrostatic terms in the model Hamiltonian, the dashed curve utilized both electrostatic and elastic strain energy terms.

It is clear that the total switchable polarization is reduced and the coercive field increased with the introduction of the elastic strain energy term. Figure 3 shows the dipole configurations at the positive (upward) and negative (downward) maximum field values in the electrostatic and elastic strain energy model.

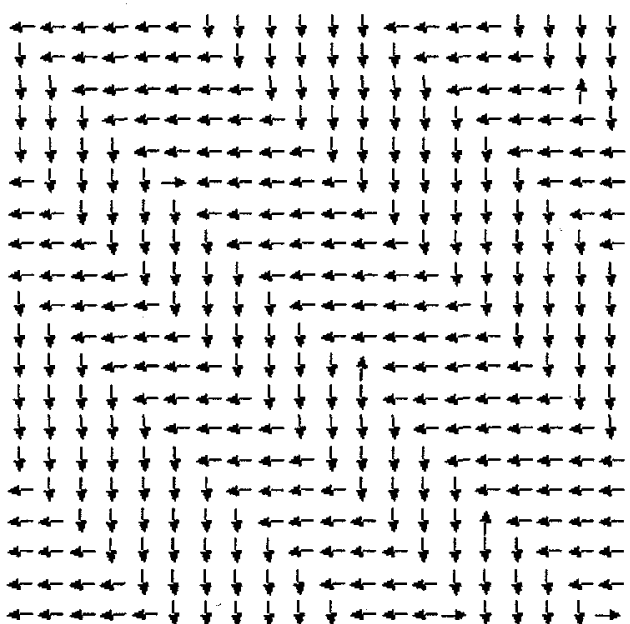

a.

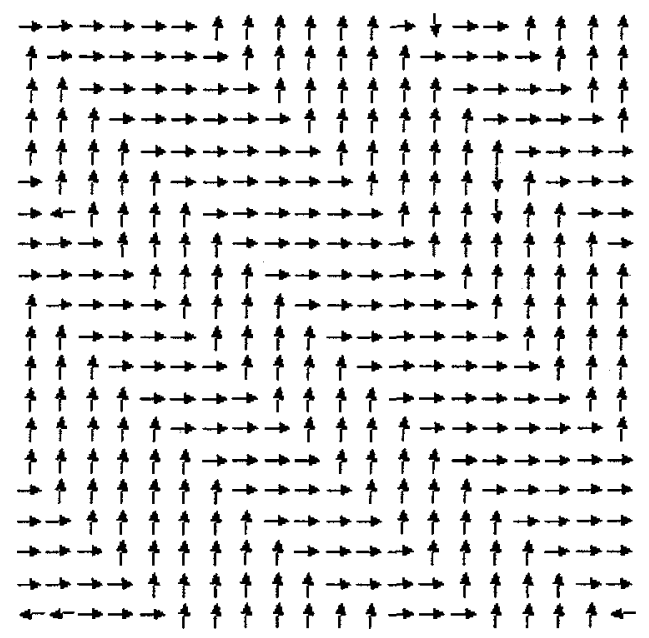

b.

FIGURE 3. Dipole configurations for the maximum negative (a.) and positive (b.) applied electric fields for the simulated hysteresis curve shown in Figure 2 for the combined electrostatic and elastic strain energy model.

Figure $4 \mathrm{a}$ depicts the $90^{\circ}$ dipole reorientation rate $\left(90^{\circ}\right.$ successes $/ 90^{\circ}$ attempts) with applied field for the combined electrostatic and strain energy run. Results for 
$180^{\circ}$ switching are given in Figure $4 \mathrm{~b}$. Analogous data for the electrostatic only case are given in Figures $5 \mathrm{a}$ and $5 \mathrm{~b}$. In all cases, the reorientation acceptance rates are initially high as the simulation begins with a randomly oriented dipole ensemble.

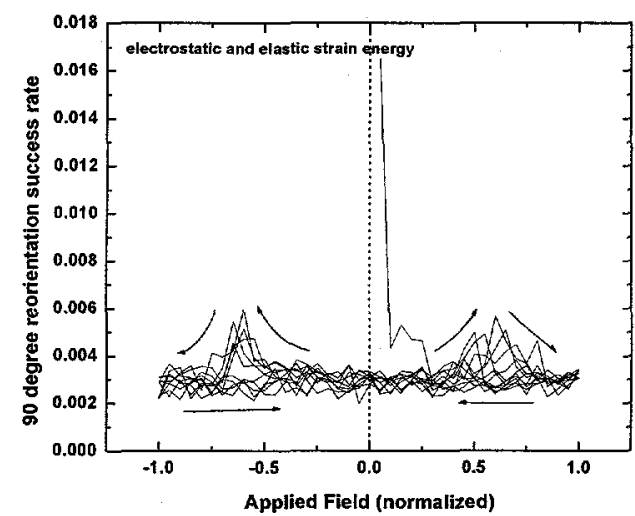

a.

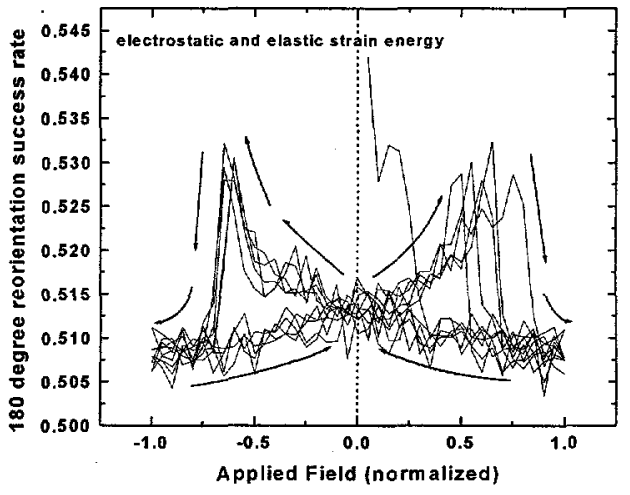

b.

FIGURE 4. Dipole reorientation rates for $90^{\circ}$ (a.) and $180^{\circ}$ (b.) reorientations corresponding to the hysteresis curve given in Figure 2 for the combined electrostatic and elastic strain energy model.

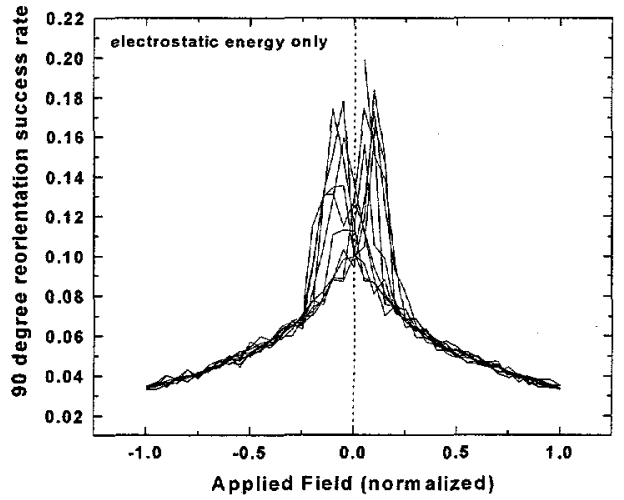

a.

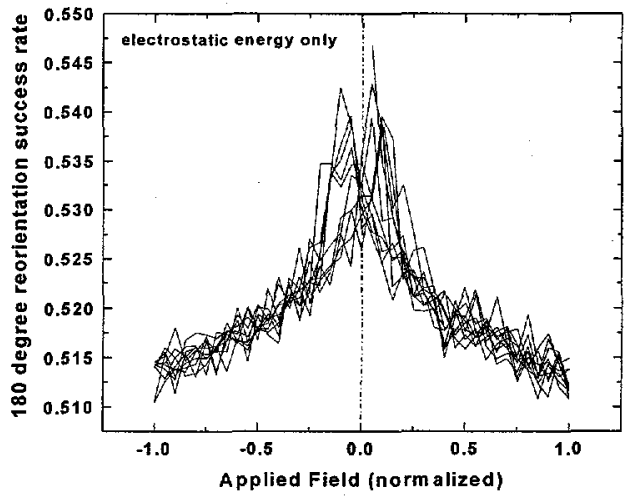

b.

FIGURE 5. Dipole reorientation rates for $90^{\circ}$ (a.) and $180^{\circ}$ (b.) reorientations corresponding to the hysteresis curve given in Figure 2 for the model containing only electrostatic energy.

In Figure 4 , the $90^{\circ}$ reorientation rate becomes nearly constant at a value only $20 \%$ of its original value and $0.6 \%$ of the corresponding $180^{\circ}$ reorientation rate at zero applied field after initial ordering of the dipoles with field early in the simulation. These consistent move success rates observed for $90^{\circ}$ reorientations are largely due to 
thermally allowed reorientations rather than a continuous switching process in the present simulation. In contrast, the $180^{\circ}$ reorientation rate is observed to gradually increase in magnitude as the positive (negative) coercive field is approached from below (above), indicating that the field-driven dipole reorientation process is occurring over a range of field magnitudes and that it is due mainly to $180^{\circ}$ switching at these field strengths. Peaks observed in both $90^{\circ}$ and $180^{\circ}$ reorientation rate curves correspond to the coercive field of the electrical hysteresis loop, a point where the maximum switching rate is expected.

A peak in the switching rates at the coercive field is also observed in the data of Figures $5 \mathrm{a}$ and $5 \mathrm{~b}$ for the electrostatic only model. In this case, however, both the $90^{\circ}$ and $180^{\circ}$ reorientation rate curves increase monotonically as the positive (negative) coercive field is approached from below (above). These observations indicate that both processes contribute to switching over a broad range of applied fields. The difference between the $90^{\circ}$ reorientation rate behaviors of the combined electrostatic and elastic strain energy model (Figure 4a) and the electrostatic only model (Figure 5a) is due to the enhanced energy penalty assigned to $90^{\circ}$ dipole reorientation when elastic strain energy is included. Significant $90^{\circ}$ reorientation success in this case is only activated when the applied field magnitude is sufficient to offset this energy penalty and increase the Monte Carlo move acceptance probability.

It is interesting to note that, while the magnitude of the $90^{\circ}$ reorientation rate is generally greater in simulations based only on electrostatic interactions (see Figure 5a), the overall success rate ratio of $90^{\circ} \mathrm{vs} 180^{\circ}$ reorientations ranges from 0.1 to 0.4 (depending on applied field) for the electrostatic-only model, indicating that, even in the absence of strain energy, $90^{\circ}$ reorientation is not the favored process for realignment with an applied field in this model.

The reduction in the switchable polarization observed in the hysteresis loop of Figure 2 (using both electrostatic and elastic strain terms), then, is the result of a limited success rate for $90^{\circ}$ dipole reorientation under even the maximum applied electric field. Examination of the dipole configurations of Figure 3, together with the reorientation rate data of Figure 4, indicate that reorientation of the dipole ensemble with the applied field is accomplished predominantly by $180^{\circ}$ switching rather than $90^{\circ}$ domain wall motion at these field strengths. It is anticipated that increased maximum field strengths will increase the $90^{\circ}$ dipole reorientation rate as an increased electrostatic driving force offsets elastic strain energy considerations for this process. This will promote the movement of $90^{\circ}$ domain walls to result in an increased saturated polarization value for the system. These issues are currently under study.

\section{CONCLUSION}

A two-dimensional, lattice-Monte Carlo model for the simulation of ferroelectric domain configurations has been presented. The model contains both electrostatic and elastic strain energy contributions to a total potential energy that determines the evolution of steady-state domain assemblages and the dynamics 
associated with reorientation of the dipole ensemble under applied electric fields. Evaluation of steady-state domain configurations obtained using purely electrostatic energy and both electrostatic and elastic strain energy terms confirm that the introduction of elastic strain considerations results in the formation of $90^{\circ}$ domain structures. Electrostatic contributions to the model favor the head-to-tail alignment of dipoles across these domain boundaries. These observations are consistent with experimental evidence in real ferroelectric materials.

The influence of elastic strain was further found to significantly impact the switching behavior observed in simulated electrical hysteresis, producing a decreased switchable polarization and increased coercive field over the model that did not include elastic strain energy. The reduced saturated polarization is attributed to a reduced $90^{\circ}$ dipole reorientation rate that precluded the long-range motion of $90^{\circ}$ domain walls under the field strengths applied.

\section{ACKNOWLEDGMENTS}

The authors thank J. Aidun, S. Hwang, and L.-Q. Chen for valuable discussions concerning the model. Sandia National Laboratories is a multiprogram laboratory operated by Sandia Corporation, a Lockheed Martin Company. This work was supported by the U.S. DOE under contract DE-AC04-94AL85000.

\section{REFERENCES}

1. W. Zhong, D. Vanderbilt, and K. M. Rabe, Phys. Rev. Lett. 73, 1861 (1994).

2. $\quad$ R.E. Cohen and H. Krakauer, Phys. Rev B 42, 6416 (1990).

3. A. Garcia and D. Vanderbilt, Appl. Phys. Lett. 72, 2981 (1998).

4. W. Cao and L.E. Cross, Phys. Rev. B 44, 5 (1991).

5. $\quad$ S. Nambu and D.A. Sagala, Phys. Rev. B 50, 5838 (1994).

6. H.-L. Hu and L.-Q. Chen, J. Am. Ceram. Soc. 81, 492 (1998).

7. M. Abel and R. Siems, Ferroelectrics 126, 275 (1992).

8. J.-M. Liu and Z.G. Liu, Mater. Lett. 36, 17 (1998).

9. B.G. Potter, Jr., V. Tikare, and B.A. Tuttle, J. Appl. Phys., to be published, 5/1/2000.

10. A.G. Khachaturyan, Theory of Structural Transformations in Solids, John Wiley and Sons, Inc., New York, NY, 1983, pp. 198-212.

11. N. Metropolis, A.W. Rosenbluth, M.N. Rosenbluth, A.N. Teller and E. Teller, J. Chem. Phys., 21, p. 1087 (1953). 